\title{
Microbiota and Obesity
}

\section{Pavel F Zabrodskii*}

Saratov Medical University "REAVIZ", Saratov, Russia

*Corresponding Author: Pavel F Zabrodskii, Saratov Medical University "REAVIZ", Saratov, Russia.
Received: October 29, 2020

Published: November 30, 2020

(C) All rights are reserved by Pavel F

Zabrodskii.
Obesity rates have increased dramatically in recent decades. Several factors involved in the etiology and pathogenesis of obesity have been identified, including diet, lifestyle, environmental factors and genetics. However, none of them fully explains the development of obesity, and the search for possible causes continues. Intestinal microbiota is considered as one of the factors influencing homeostasis (regulation of the level of basic metabolism depending on the diet) [1] through several supposed mechanisms studied on experimental models and humans (synthesis of shortchain fatty acids, metabolic endotoxemia, role of endocannabinoid receptor system, participation of microbiota in lipogenesis, regulation of appetite by microbiota production of short-chain fatty acids and modulation of synthesis of certain peptides by intestinal enteroendocrine L-cells, inhibition of fatty acid oxidation).

There is reason to believe that mammals with obesity should have a microbial composition in the intestines that is different from microbiota in skinny representatives of this class of vertebrates. Studies on mice have shown higher percentages of Firmicutes in the intestines and a simultaneous decrease in Bacteroidetes in fat mice [2]. Studies in humans have shown contradictory results. Both a high Firmicutes/Bacteroidetes ratio and an opposite ratio of these microbiota representatives were found [3]. A lower relative number of Bacteroidetes in the intestine compared to the number of Aktinobacteria in obese people than in skinny people was proved, without significant differences in the content of Firmicutes [4]. A number of studies show that obesity is associated with a relatively high content of Bacteroidetes, Firmicutes, M. smithii, E. coli and various Lactobacillus species in the intestines. There is a direct correlation between the intestinal content of L. reuteri and obesity, where higher L. reuteri content and lower L. casei/L. paracasei and L. plantarum levels are associated with a high body mass index [5]. There is a feedback between intestinal content in Bifidobacterium and obesity [3]. Similar data were obtained by studying the relationship between obesity and the intestinal content of Akkermansia muciniphila, as well as $C$. perfingens and C. bacteroidetes [6]. A new microbial biomarker of obesity, Christensenellaceae, has been proposed; this microorganism contributed to the reduction of body weight in mice [7].

The question of which "indicator" microbial group is responsible for obesity is relevant, as there are many contradictory results regarding the presence or absence of a specific microbiota in obesity. These discrepancies may be related to the human genetic background, age, gender, geographic location and diversity of the intestinal microbiota. Determining the potential role of the intestinal microbiota will help in the future identify the biomarkers of obesity and metabolic changes. Remodeling intestinal microbiota may change the metabolic status of obese patients, in particular the transition from metabolically unhealthy obesity to metabolically neutral. Modulation of intestinal microbiota using pre and probiotic drugs may be one of the possible strategies to prevent and treat obesity.

\section{Bibliography}

1. Kasubuchi M., et al. "Dietary gut microbial metabolites, shortchain fatty acids, and host metabolic regulation". Nutrients 7.4 (2015): 2839-2849. 
2. Aron-Wisnewsky J., et al. "Gut microbiota and non-alcoholic fatty liver disease: new insights". Clinical Microbiology and Infection 19.4 (2013): 338-348.

3. Schwiertz A., et al. "Microbiota and SCFA in lean and overweight healthy subjects". Obesity 18.1 (2010): 190-195.

4. Turnbaugh PJ., et al. "A core gut microbiome in obese and lean twins". Nature 457.7228 (2009): 480-484.

5. Million M., et al. "Correlation between body mass index and gut concentrations of Lactobacillus reuteri, Bifidobacterium animalis, Methanobrevibacter smithii and Escherichia coli". International Journal of Obesity 37.11 (2013): 1460-1466.

6. Dahiya DK., et al. "Gut Microbiota Modulation and Its Relationship with Obesity Using Prebiotic Fibers and Probiotics: A Review". Frontiers in Microbiology 8 (2017): 563.

7. Goodrich JK., et al. "Human genetics shape the gut microbiome". Cell 159.4 (2014): 789-799.

\section{Assets from publication with us}

- Prompt Acknowledgement after receiving the article

- Thorough Double blinded peer review

- Rapid Publication

- Issue of Publication Certificate

- High visibility of your Published work

Website: https://www.actascientific.com/

Submit Article: https://www.actascientific.com/submission.php

Email us: editor@actascientific.com

Contact us: +919182824667 